\title{
Metaphyseal chondrodysplasia
}

\author{
J. W. SPRANGER \\ M.D. \\ Children's Hospital, Johannes Gutenberg University, Mainz
}

\begin{abstract}
Summary
Any review of the metaphyseal chondrodysplasias is complicated by their variety and mainly unknown pathogenesis. The more familiar types display considerable clinical and radiological diversity: even more so the rarer disorders which still require complete definition, but differences in their mode of inheritance make diagnostic precision mandatory. These dysplasias present in infancy or in childhood, when the patient, usually dwarfed, may be proportionate, so that some forms may be confused with rickets or other lesions. Mental retardation is unusual, but the skin, hair, nails and facies provide valuable diagnostic features.

Radiological abnormalities mainly affect the metaphyses of the shortened limb bones, less often the skull, vertebrae, pelvis, ribs and extremities, and sometimes their distribution may indicate the specific type of dysplasia. In a further complex group multiple systems are involved, notably the pancreas, intestine and lympho-reticular, causing malabsorption and haematological or immunological disorders.
\end{abstract}

THE various metaphyseal chondrodysplasias are intrinsic disorders of skeletal development, characterized by abnormal radiographic appearances and, in most cases, by a disturbed histological structure of the metaphyses, the cause of which is unknown. The epiphyses and the vertebrae are only minimally affected. Their intrinsic nature implies that these conditions are due to a defective growth potential of cartilage and bone. They must be carefully distinguished from acquired secondary metaphyseal lesions such as those due to rickets, scurvy, trauma, etc., in which the growth potential of cartilage and bone is initially quite normal. These secondary metaphyseal changes are not included in the group under review and, by convention, achondroplasia and hypochondroplasia are excluded, although both are radiologically and histologically somewhat

Correspondence: Professor J. W. Spranger, Children's Hospital, Johannes Gutenberg University, 6500 Mainz, Germany. similar and might also be classified as metaphyseal disorders.

(1) Commoner forms of metaphyseal chondrodysplasias (Table 1)

Hypophosphatasia

This is the only metaphyseal chondrodysplasia of known pathogenesis, being caused by a deficiency of alkaline phosphatase, an enzyme which, in a simplistic description, behaves as a pyrophosphatase, destroying pyrophosphate which itself inhibits tissue mineralization. In the absence or deficiency of the enzyme, pyrophosphate accumulates and interferes with normal tissue calcification. This phosphatase deficiency is variable in degree.

TABLE 1. The metaphyseal chondrodysplasias

1. Well defined forms
Hypophosphatasia
Jansen type
Schmid type
Vaandrager-Peña type

2. Rarer forms (not well defined)

Rimoin \& McAlister (1971)

Van Creveld et al.

Wiedemann \& Spranger (1970)

Kozlowski (1964)

Rosenblum \& Smith (1965)

Spahr \& Spahr-Hartmann (1961)

3. With multi-system involvement

Metaphyseal chondrodysplasia with malabsorption and neutropenia (Schwachman-Diamond syndrome)

McKusick type (cartilage hair hypoplasia)

Adenosine deaminase deficiency

Metaphyseal chondrodysplasia with humoral immunodeficiency

Severe forms of hypophosphatasia are incompatible with life, perinatal death occurring in days, less often months, from respiratory failure. Less severely affected patients may survive for varying periods, but hypercalcaemia gradually develops after the first weeks of life with failure to thrive, episodic bouts of constipation, fever and nephrocalcinosis. If the infant survives the first year, 
spontaneous improvement takes place. Craniostenosis may occur with premature shedding of teeth and these may be the only clinical symptoms in mild cases. Microscopically, the only changes noted are the tongues of unmineralized cartilage or osteoid extending into the shafts of the long bones.

It is important to note that the congenital lethal and the milder adult tarda forms have been observed in a single family and are hence expressions of a single gene mutation (Macpherson, Kroeker and Houston, 1972). The range of genetic variability is quite remarkable for an autosomal recessive condition, simulating that reported by Siggers (1977) for cartilage hair hypoplasia.

Both liver and bone alkaline phosphatase are lowered, whilst the intestinal alkaline phosphatase may be either normal or elevated. Clinically normal heterozygotes can be identified by a slightly reduced serum alkaline phosphatase and may also have increased urinary excretion of phosphoethanolamine. It is important to note that the clinical and radiographic features of hypophosphatasia have been observed in patients with a normal level of blood alkaline phosphatase. This has been described as 'pseudo-hypophosphatasia', but there is at least one family described in which the various features of hypophosphatasia occurred with normal or low levels of serum alkaline phosphatase (Méhes et al., 1972). Thus, so-called 'pseudo-hypophosphatasia' may not be a nosological entity.

\section{Metaphyseal chondrodysplasia-Jansen type}

This is well known and is inherited as an autosomal dominant. The original patient reported by Jansen (1934) has been re-examined, and at 44 years of age is stated to be in a remarkably good state of health and running a drug store (De Haas, De Boer and Griffioen, 1969).

With advancing age the metaphyseal abnormalities tend to disappear with fusion of the epiphyses, but the bones remain short and deformed (Fig. 1). The early skull changes consist of a peculiar reticular pattern of the calvaria. Later there is a severe degree of sclerosis with hyperostosis of the skull base, but the mandible may be small and there may be persistent metaphyseal irregularities. One case reported by Ozonoff (1969) was described with these changes but did not have the Jansen type of metaphyseal chondrodysplasia, so that the early metaphyseal appearances should not be regarded as entirely diagnostic.

\section{Metaphyseal chondrodysplasia-Schmid type}

This well-defined disorder is also transmitted as an autosomal dominant, although in some patients who are children of clinically and radiologically normal parents, a recessive inheritance must be

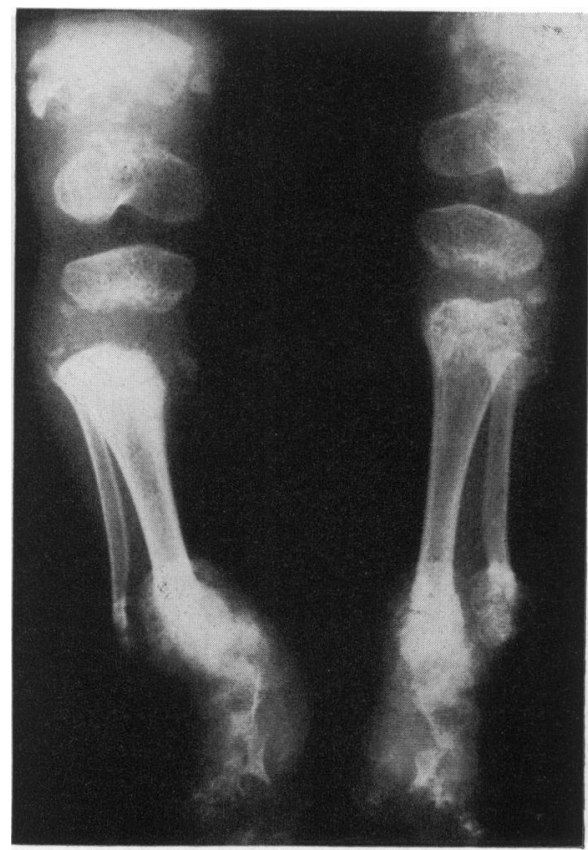

FIG. 1. Metaphyseal chondrodysplasia, Jansen type. The tubular bones are severely shortened with striking metaphyseal irregularities, normal appearing epiphyses and very wide physes. There are no major differential diagnostic problems.

considered possible. The Schmid type is commoner than the Jansen type, and at least fifty-one cases have been reported since the original paper by Schmid in 1949. In the Mainz Bone Dysplasia Register there are nine cases of the Schmid type to only three of the Jansen type.

This is a comparatively benign condition without major complications, with a normal life span and an adult height of $130-160 \mathrm{~cm}$. Its main clinical significance is that it may be confused with, and sometimes treated as vitamin D-resistant rickets.

The most striking radiographic abnormalities are well marked metaphyseal changes in the proximal rather than the distal femora (Fig. 2)-quite the opposite of the distribution found in cartilage hair hypoplasia (Fig. 3). Furthermore, in the Schmid type there is much less coxa vara and osteotomies are inadvisable as the spontaneous prognosis is very favourable.

As mentioned above, the radiological changes of metaphyseal chondrodysplasia may be closely simulated by rickets (Fig. 4) and to a lesser degree by scurvy (Fig. 5) or by traumatic damage (Fig. 6) including the so-called 'battered child'. 


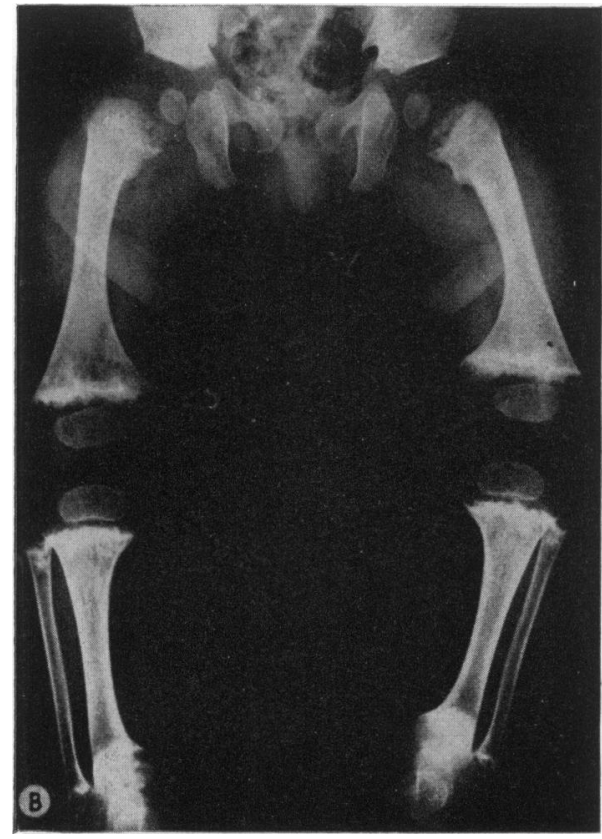

FIG. 2. Metaphyseal chondrodysplasia, Schmid type. The metaphyscal changes are as severe in the proximal as in the distal femora. The femoral necks are in varus position. Both fcaturcs help to differentiate the Schmid from the McKusick type (cartilage hair hypoplasia) of metaphyseal chondrodysplasias. The changes in this patient are rather marked; they may be milder in other instances.

\section{Metaphyseal chondrodysplasia-Vaandrager type}

This disorder is fairly well defined, although very rare, and first described by Vaandrager (1960) who reported monozygous twins with peculiar metaphyseal changes. Radiographically, there are longitudinal radiolucent areas extending deeply into the diaphyses, the ends of the more severely afflicted bones being expanded. The spine is normal, and the severity of the long bone changes is again variable. Peña (1965) described siblings with similar but more severe changes, together with scoliosis and defects of the cervical vertebrae. In a girl patient the author noted that the bodies of the cervical vertebrae were flattened, whilst those of her 14-year-old brother were uncalcified, these features being absent in Vaandrager's patients. The knuckles were small, but radiographs of the hands were stated to be normal.

Another patient with mild metaphyseal chondrodysplasia and slightly flattened vertebral bodies has been described by Kozlowski and Sikorska (1970). With our present knowledge and information it is uncertain whether the differences between these various cases are due to expression of a second mutation, or whether there is an autosomal dominant

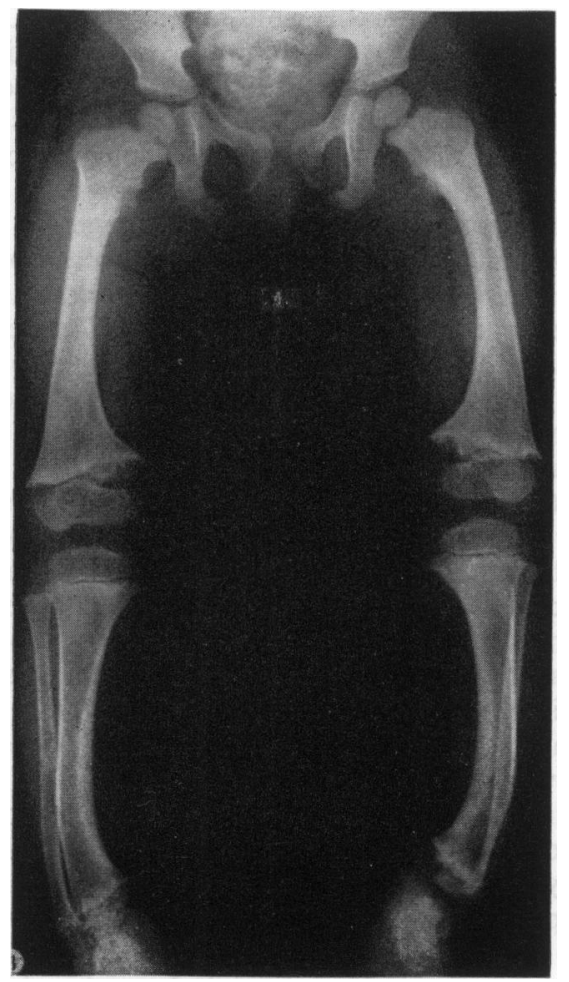

FIG. 3. Metaphyseal chondrodysplasia, McKusick type (cartilage hair hypoplasia). The metaphyseal changes are more pronounced in the distal than in the proximal femora.

Vaandrager type with a normal spine and also an autosomal recessive or hump-backed variant with distinct spinal changes.

(2) Metaphyseal chondrodysplasia-other rarer types (Table 1)

These appear to be nosological entities, but as yet not adequately described.

\section{Rimoin and McAlister (1971)}

In 1971 Rimoin and McAlister described three brothers, offsprings of a consanguineous mating, with short-limbed dwarfism, conductive hearing loss, mental retardation and a metaphyseal dysplasia more severe in the middle and distal segments of the limbs than in the proximal. Similar bone changes were described by Kozlowski and Rupprecht (1972) from a 7-year-old boy who was mentally normal and no hearing defect was mentioned.

Van Creveld et al. (1971)

Van Creveld et al. (1971) described two unrelated 


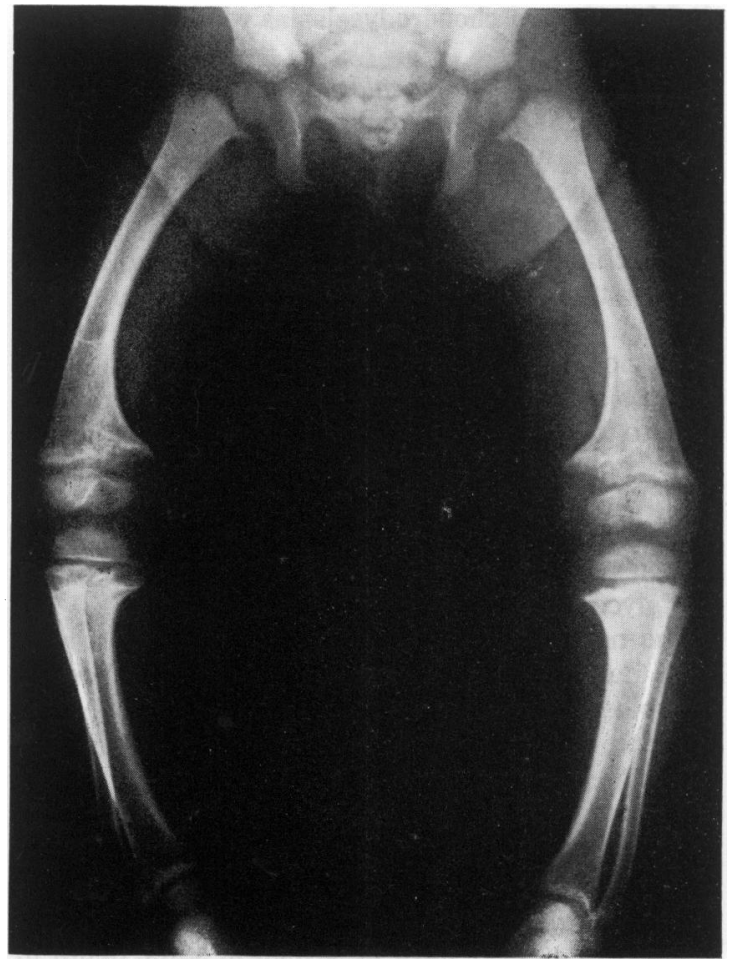

FIG. 4. Hypophosphataemic rickets. Note the striking similarity of the bone changes to those in the metaphyseal chondrodysplasias. The major radiographic difference is the abnormal bone structure in rickets. Vitamin $D$ and phosphate therapy is required in rickets but could be lethal in the chondrodysplasias.

patients with a severe disorder resembling the Jansen type of chondrodysplasia. In contrast to the Jansen type, however, there were large enchondromatous lesions extending into the ear bones. In the later stages of evolution, these defects were studded with fine calcifications. There is some resemblance to multiple enchondromatosis, but the condition is more symmetrical and more severe than the usual case of Ollier disease.

\section{Wiedemann and Spranger (1970)}

Another form of metaphyseal chondrodysplasia was described by Wiedemann and Spranger (1970) with undoubtedly severe metaphyseal changes in the long bones tending to disappear with advancing age and terminating, in contrast with other types of metaphyseal chondrodysplasia, with a normal stature. The vertebrae have separate ossification centres at birth; later they fuse, the changes regress and the spine eventually appears radiologically normal. There is one more case in the literature

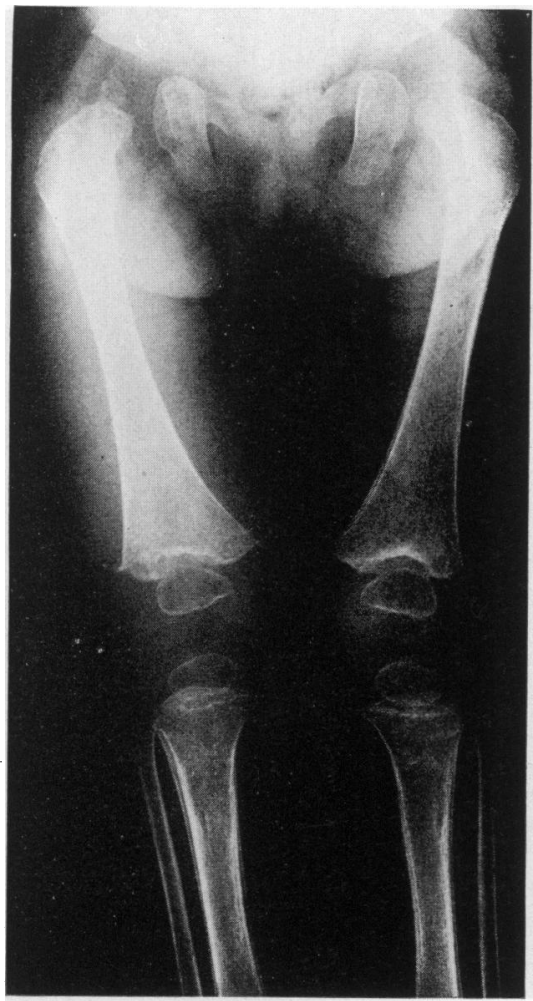

Fig. 5. Scurvy. The metaphyseal changes could be confused with those in the chondrodysplasias or those in battered children. A major differential criterion is the abnormal bone structure in scurvy.

(Wiersbitzky, Weyrauch and Wiersbitzky, 1970) and it appears to be an entity.

\section{Kozlowski (1964)}

Another condition was reported by Kozlowski (1964) who described a family of five siblings and two unrelated children with short stature. The radiographic abnormalities were so mild that Kozlowski required to add arrows to his illustrations to demonstrate them. Possibly there are forms of metaphyseal chondrodysplasia that are extremely mild.

\section{Rosenbloom and Smith (1965)}

One large family is often cited as a paradigm of the Schmid type with autosomal dominant transmission; but the metaphyseal changes at the knees are more severe than those in the proximal femora, a reversal of the usual distribution of the Schmid type (Rosenbloom and Smith, 1965). In this family, on careful examination, epiphyseal changes may 


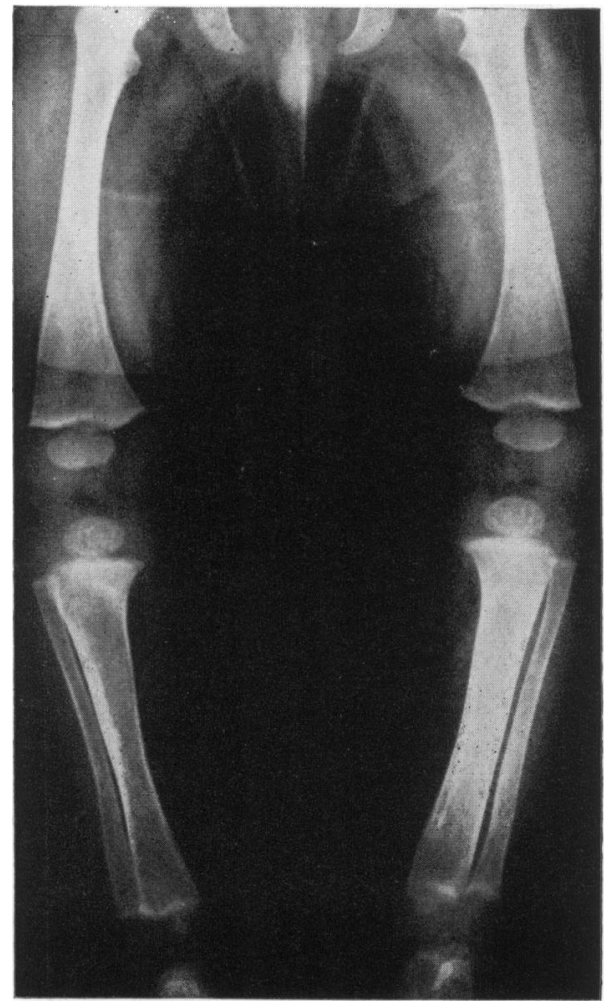

FIG. 6. Menkes syndrome. Metaphyseal irregularities are seen in the tibia and distal fibula. The left tibial shaft is expanded owing to an old periosteal haematoma with secondary bone formation. The appearance of these bones is more likely to be confused with those of a battered child than with those of a metaphyseal chondrodysplasia.

also be noted, but no coxa vara as might be expected in the conventional Schmid type. Rather, there is coxa valga with flattening of the femoral heads. In this family the correctness of the diagnosis of Schmid type metaphyseal chondrodysplasia seems questionable and possibly these patients may have arthro-ophthalmopathy described by Stickler et al (1965) as some had myopia and cleft palates.

\section{Spahr and Spahr-Hartmann (1961)}

Two brothers were reported by Spahr and Spahr-Hartmann (1961) in Switzerland who had metaphyseal abnormalities which were hard to classify. The older sibling had severe constipation, probably caused by an aganglionic megacolon and died from oesophageal haemorrhage due to severe thrombocytopenia. The question arises that these may have been cases of cartilage hair hypoplasia.
(3) Metaphyseal chondrodysplasias with multi-system defects (Table 2)

Metaphyseal chondrodysplasia, malabsorption, neutropenia (MMN-) syndrome

To date, about seventeen cases have been reported. Since 1964 it has sometimes been referred to as the 'Schwachman-Diamond syndrome' (Schwachman et al., 1964). The metaphyseal defects were first noted by Burke et al. (1967) and are most marked in the proximal femora. Unlike other forms of metaphyseal chondrodysplasias, these changes are due to failure of the columnar cartilage cells to hypertrophy. Electron microscopic studies have shown a peculiar accumulation of abnormal intracellular material with finger-print-like patterns in the rough endoplasmic reticulum of the chondrocytes (Spycher et al., 1974). This may consist of a glycoprotein but is still undefined.

The neutropenia in this disorder is unexplained; some data point to a maturation arrest, others to hypoplasia of the neutrophil precursors. No definite immune defect has been demonstrated in this syndrome, but recurrent respiratory infections are quite common although not related to the severity of the neutropenia.

\section{Cartilage hair hypoplasia}

This has already been described in some detail in the preceding paper by Dr Siggers et al. (1977). The disorder may be associated with defects of the intestine, the blood cells, the immune system and the hair (Table 2). In the newborn the first sign is bowing of the femora which is diagnostic, with metaphyseal changes only apparent after 4 months of age. The original paper by McKusick et al. (1965) reported six of seventeen patients with intestinal malabsorption and two with congenital megacolon.

TABLE 2. Metaphyseal chondrodysplasia with multi-system involvement

\begin{tabular}{lcccc}
\hline & MMN & CHH & ADAD & MHD \\
\cline { 2 - 5 } Malabsorption & + & + & + & - \\
Hirschsprung disease & - & + & - & - \\
Erythropenia & + & + & + & - \\
Neutropenia & + & + & + & - \\
Thrombocytopenia & + & - & - & - \\
Lymphopenia & - & + & + & - \\
Cellular immunodeficiency & - & + & + & - \\
Humoral immunodeficiency & - & - & + & + \\
Hair changes & + & + & + & - \\
Skin changes & - & - & + & - \\
\hline
\end{tabular}

MMN, Metaphyseal chondrodysplasia, malabsorption, neutropenia; $\mathrm{CHH}$, cartilage hair hypoplasia; ADAD, adenosine deaminase deficiency; MHD, metaphyseal chondrodysplasia, humoral immunodeficiency; - + , reported - not reported. 
This combination of metaphyseal chondrodysplasia and intestinal abnormalities has been confirmed by others.

Haematological changes have often been reported, e.g. gross neutropenia due to myeloid arrest at the metamyelocyte level with lymphopenia. Two cases were reported in the French literature by Sacrez et al. (1965) and L'Hirondel et al. (1967) of congenital aplastic anaemia in cartilage hair hypoplasia. The marrow defect involves both the red and white cell systems.

In some cases, there is defective cellular immunity which explains the increased susceptibility to viral infections, notably to chickenpox and smallpox vaccination. Lymphoid cell transformation and delayed hypersensitivity to Candida are normal in some patients, abnormal in others. No skin abnormality has been reported in cartilage hair hypoplasia, but the hair is usually fine and sparse.

\section{Adenosine deaminase deficiency}

This is the first of this group of dysplasias with a pathogenetic explanation as to why so many systems -cartilage cells, immune and haematopoietic tissues-are involved. The association of combined $B$ and $T$ cell immunodeficiency with metaphyseal chondrodysplasia was first observed by McKusick and Cross (1966), but at that time was thought to be co-incidental. Further reports, however, showed that the association was pathogenetically relevant. It has recently been demonstrated that infants and young children with combined immunodeficiency and metaphyseal chondrodysplasia had invariably a deficiency of the enzyme adenosine deaminase in their erythrocytes (Meuwissen, Pollara and Pickering, 1975). Bone changes, however, were only noted in patients with severe immunodeficiency, but not in those with some preservation of B or $\mathrm{T}$ cell function. The clinical picture of severe adenosine deaminase deficiency is characterized by failure to thrive, chronic diarrhoea with malabsorption, chronic or recurrent pneumonia, candidiasis and/or opportunistic infections and short stature. The skin is thick and redundant, with scaling of the scalp and sparse body hair (Table 2).

In neonates and young infants, radiographs show an abnormally shaped pelvis, the wings of the ilia being low and broad. There are metaphyseal changes and conspicuous flaring and cupping of the costochondral junctions.

Adenosine deaminase which is normally present in various tissues including liver, muscle and intestinal mucosa, is involved in purine metabolism, converting adenosine to inosine. Possibly, if the enzyme is absent, adenosine may accumulate within cells and may suppress normal maturation or inhibit the proliferation of lymphoid, cartilage and maybe other cells.

Metaphyseal chondrodysplasia and humoral immunodeficiency

A case recently shown to the author by Professor C. O. Carter had metaphyseal chondrodysplasia and deficient humoral immunity but normal cellular immunity. This occurrence was first described by Ammann, Sutliff and Millinchick (1974) who reported a patient with a bone dysplasia, normal cellular and defective humoral immunity. One further case was studied by Dr J. Herrmann in Madison, Wisconsin.

Thus it appears that all three possibilities exist: metaphyseal chondrodysplasia with cellular, humoral or combined immunodeficiency. Further studies of the nucleic acid metabolism may be rewarding in these conditions.

\section{References}

AmmanN, A.J., Sutliff, W. \& Millinchick, E. (1974) Antibody-mediated immunodeficiency in short-limbed dwarfism. Journal of Paediatrics, 84, 200.

Burke, V., Colebatch, J.H., Anderson, C.M. \& Simons, M.J. (1967) Association of pancreatic insufficiency and chronic neutropenia in childhood. Archives of Disease in Childhood, 42, 147.

De HaAs, W.H.D., De Boer, W. \& Griffioen, F. (1969) Metaphyseal dysostosis. Journal of Bone and Joint Surgery, 51B, 290.

JANSEN, M. (1934) Über atypische Chondrodystrophie (Achondroplasie) und über eine noch nicht beschriebene angeborene Wachstumstörung des Knochensystems. Zeitschrift für orthopädische Chirurgie, einschliesslich der Heilgymnastik und Massage, 61, 253.

Kozlowski, K. (1964) Metaphyseal dysostosis. Report of five familial and two sporadic cases of a mild type. American Journal of Roentgenology, Radiation Therapy and Nuclear Medicine, 91, 602.

KozlowsKi, K. \& RupPrecht, E. (1972) Metaphyseal dysplasia with peripheral location. Helvetica paediatrica acta, 27, 85.

Kozlowski, K. \& Sikorska, B. (1970) Dysplasia metaphysaria, Typ Vaandrager-Peña. Zeitschrift für Kinderheilkunde, 108, 165.

L'Hirondel, J., Cane, Daridon, \& Tillet, (1967) Anémie de Blackfan-Diamond et dysostose métaphysaire récessive autosomique. Ouest Médical, 20, 1152.

McKusiCK, V.A. \& CROss, H.E. (1966) Ataxia-telangiectasia and Swiss type agammaglobulinemia. Journal of the American Medical Association, 195, 739.

McKusick, V.A., Eldridge, R., Hostetler, J.A., RuangWIT, O. \& Egeland, J.A. (1965) Dwarfism in the Amish, II. Cartilage hair hypoplasia. Bulletin of the Johns Hopkins Hospital, 116, 285.

Macpherson, R.I., Kroeker, M. \& Houston, C.S. (1972) Hypophosphatasia. Journal of the Canadian Association of Radiologists, 23, 16.

Maroteaux, P., Savart, P., Lefebvre, L. \& Royer, P. (1963) Les formes partielles de la dysostose métaphysaire. Presse Médicale, 71, 1523.

Méhes, K., Klujber, L., Lassu, G. \& Kajtár, P. (1972) Hypophosphatasia: screening and family investigations in an endogamous Hungarian village. Clinical Genetics, 3, 60. 
Meuwissen, H.J., Pollara, B. \& Pickering, R.J. (1975) Combined immunodeficiency disease associated with adenosine deaminase deficiency. Journal of Pediatrics, 80, 1010.

Ozonoff, M.B. (1969) Metaphyseal dysostosis of Jansen. Radiology, 93, 1047.

Peña, J. (1965) Disostosis metafisaria. Una revision con apertacion de una observacion familiar, una forma nueva de la enfermedad. Radiologica. Madrid, 47, 3.

Rimoin, D.L. \& McAlister, W.H. (1971) Metaphyseal dysostosis, conductive hearing loss and mental retardation: a recessively inherited syndrome. Birth Defects, 7, 116.

Rosenbloom, A.L. \& SMith, D.W. (1965) The natural history of metaphyseal dysostosis. Radiology, 83, 665.

SACrez, R., LeVy, J.M., Godar, G. \& Castanier, J. (1965) Anémie de Blackfan-Diamond associé à des malformations multiples. Médecine Infantile, 72, 493.

SchмID, F. (1949) Beitrag zur Dysostosis Enchondralis Metaphysaria. Monatsschrift für Kinderheilkunde, 97, 393.

Schwachman, H., Diamond, L.K., Oski, F. \& Kon-T KhaW (1964) The syndrome of pancreatic insufficiency and bone marrow dysfunction. Journal of Pediatrics, 65, 645.

Siggers, D.C., Burke, J.B., Morris, B., Normand, I.C.S., Tanner, J.M. \& Williamson, D.A.J. (1977) Cartilage hair hypoplasia. Postgraduate Medical Journal, 53, 473.
SPahr, A. \& SPahr-Hartmann, I. (1961) Dysostose métaphysaire familiale. Étude de 4 cas dans une famille. Helvetica paediatrica acta, 16, 834.

SPyCher, M.A., Giedion, A., SChMerling, D.H. \& RüTtNER, J.R. (1974) Electron microscopic examination of cartilage in the syndrome of exocrine pancreatic insufficiency, neutropenia, multiple dysostosis and dwarfism. Helvetica paediatrica acta, 29, 471.

Stickler, G.B., Belau, P.G., Farrell, F.J., Jones, J.D., Pugh, D.G., Steinberg, A.G. \& WARD, L.M. (1965) Hereditary progressive arthro-ophthalmopathy. Mayo Clinic. Proceedings, 40, 433.

VAANDRAGER, G.J. (1960) Metafysaire dysostosis. Nederlands Tijdschrift voor Geneeskunde, 104, 547.

van Creveld, S., Kozlowski, K., Pietron, K. \& van der VALK, A. (1971) Metaphyseal chondrodysplasia calcificans. A report on two cases. British Journal of Radiology, 44, 773.

Wiedemann, H-R. \& Spranger, J.W. (1970) Chondrodysplasia metaphysaria (Dysostosis metaphysaria) ein neuer typ. Zeitschrift für Kinderheilkunde, 108, 171.

Wiersbitzky, H., WeyraUch, P.C. \& Wiersbitzky, S. (1970) Dysostosis enchondralis metaphysaria (Typ Schmid) bei einem Neugeborenen mit Morbus haemolyticus neonatorum (Anti-D). Deutsches Gesundheitswesen, 25, 2225. 